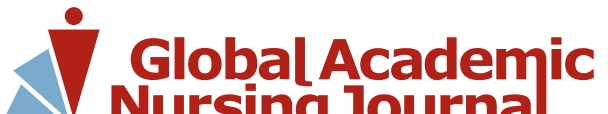 Nursing Journal
}

\section{Cardiologia, suas interfaces e a importância da interdisciplinaridade}

Cardiology, its interfaces and the importance of interdisciplinarity

Cardiología, sus interfaces y la importancia de la interdisciplinariedad

\begin{abstract}
Juliana Mendes Marques ${ }^{1}$
ORCID: 0000-0002-6730-9649

${ }^{1}$ Universidade Federal do Estado do Rio de Janeiro. Rio de Janeiro, Brasil.
\end{abstract}

Como citar este artigo:

Marques JM. Cardiologia, suas

interfaces e a importância da interdisciplinaridade. Glob Acad Nurs. 2021;2(Spe.3):e160.

https://dx.doi.org/10.5935/26755602.20200160

Autor correspondente: Juliana Mendes Marques E-mail: jumendesm@hotmail.com

Editor Chefe: Caroliny dos Santos Guimarães da Fonseca Editor Executivo: Kátia dos Santos Armada de Oliveira

Submissão por convite: 14-09-2021
As Ciências em Saúde vêm avançando cada vez mais no âmbito científico. As formações acadêmicas estimulam a produção cientifica para que haja um olhar cada vez mais centralizado, seja de forma individualizada ou coletiva sobre determinado sujeito ou população estudada. No âmbito da Cardiologia, em particular, dados epidemiológicos mostram que as doenças cardiovasculares são a principal causa de mortalidade e morbidade em todo mundo ${ }^{1}$, trazendo, portanto, a importância de se ter um olhar biopsicossocial para com o indivíduo, levando o processo avaliativo para além das competências de conhecimento técnico.

Nesse contexto, o processo avaliativo abarca o conhecimento, o discernimento de evidências, a habilidade técnica, o raciocínio clínico, das emoções, dos valores, do uso adequado da comunicação, e a reflexão na prática diária para o benefício de indivíduos e da comunidade à qual ele serve.

A Cardiologia não atende mais somente o modelo hospitalocêntrico, intervencionista e curativo, onde procedimentos invasivos e farmacológicos eram levados como a "verdadeira" medicina. Um exemplo claro é o momento em que o mundo está vivendo: a valorização da saúde mental. Há cada vez mais relatos de crises de ansiedade que são constantemente confundidas com algum diagnóstico cardíaco.

Para além disso, é importante saber que indivíduos que possuem diagnósticos de transtornos mentais tem um fator de risco aumentado para o adoecer cardíaco. O profissional de saúde que trabalha com a cardiologia precisa de um olhar atento para todas as questões que envolvem o ser humano, iniciando no discurso do conhecimento teórico, concomitantemente ao desenvolvimento de habilidades, pensando sempre na multidisciplinaridade e interdisciplinaridade.

Na edição em questão, as produções são relacionadas a estudos voltados para a cardiologia, abrangendo todos os níveis de atenção em saúde. As metodologias elucidadas proporcionarão uma análise ampla sobre o tema, trazendo informações atuais e relevantes para o meio científico.

O diagnóstico cardíaco precisa ser avaliado com cautela, entendendo sempre a etiologia da clínica apresentada, se há alterações anatômicas e fisiológicas e incapacidade funcional.

Mas é importante salientar que o corpo não pode ser segmentado e que doenças de etiologias não cardíacas podem ocasionar sintomas cardíacos, 
ou até mesmo, doenças cardíacas. Por fim, a Cardiologia proporciona uma ampla discussão multidisciplinar, abrangendo toda interface das áreas de saúde e conectando o corpo à subjetividade da mente e exaltando sempre a soberania da clínica.

\section{Referências}

1. Nascimento BR, Brant LCC, Oliveira GMM, Malachias MVB, Reis GMAl, Teixeira RA, et al. Cardiovascular Disease Epidemiology in Portuguese-Speaking Countries: data from the Global Burden of Disease, 1990 to 2016. Arq Bras Cardiol. 2018;110(6):500-511. DOI: $10.5935 / A B C .20180098$ 